\title{
Scanning the welded joints of aluminium alloys using subminiature eddy-current transducers
}

\author{
Sergey Dmitriev ${ }^{1, *}$, Alexey Ishkov ${ }^{2}$, Vladimir Malikov ${ }^{1}$, and Anatoly Sagalakov ${ }^{1}$ \\ ${ }^{1}$ Altai State University, 656049 Lenina Av., Barnaul, Russia \\ ${ }^{2}$ Altai State Agricultural University, 656049 Krasnoarmeyskiy Av., Barnaul, Russia
}

\begin{abstract}
Aluminium has a reputation for ease of use, strength and durability. In addition to its exceptional aesthetic properties, solid aluminium does not burn. As architects, contractors, consultants and real estate owners look to meet stringent safety requirements in the construction and refurbishment of high-rise constructions for both residential and commercial uses, aluminium cladding provides an alternative that is not only safe but that is also durable and attractive. One of the ways to connect elements into a aluminium construction is welding. friction stir welding is one of the most efficient. The authors developed a measuring system based on subminiaturized eddy-current transducers aimed at examining locally the defects of welded joints in aluminium-magnesium alloy plates connected by means of friction stir welding. The authors made a modification of the Delyann filter, which allowed them to increase considerably the signal-noise relations. The dependency of the eddy-current transducer response on defects was provided, i.e. concealed cuts and openings inside the welded joint, at the frequencies of $100-10000 \mathrm{~Hz}$ of the exciting winding.
\end{abstract}

\section{Introduction}

Constructions made of aluminium alloys have got a frequent practice in current technology. Application of these alloys in such spheres as aircraft engineering, high-rise constructions, automobile construction, spacecraft engineering, demands higher standards of the structure and mechanical characteristics of the material. One of the ways to connect elements into a construction is welding. However, common types of fusion welding are not always applicable for some aluminium-based alloys. In order to join elements of such alloys, one has to use special methods and processing technologies, among which, friction stir welding is one of the most efficient $[1,2]$.

Due to significant temperature and deformity gradients, obtained welded joints are characterized by high inhomogeneity and deficiency of the structure. Affected by external mechanical impact, structural defects of the welded joints cause early crack formation and destruction. As soon as the obtained constructions can be used in extreme operation conditions, the mentioned circumstance requires the performance of welded joints thorough diagnosis. Currently, friction stir welding (FSW) is widely applied in the production of body frame elements made of aluminium alloys. This method allows us to get permanent

* Corresponding author: osys@me.com 
qualitative joints even made of those alloys that are not subject to electric-arc welding or argon-arc welding. The ultrasonic technique with the use of phased array antennas demonstrates capability of detecting defects within the whole volume of the weld pass. The obtained results are highly valid. However, except for defects, located within the joint volume, surface defects, such as bridging, interstices and cracks in the surface layer and others, influence the joint strength substantially. One of the most highly-efficient and exact methods, which allows detecting defects located in the surface or in the thin subsurface layer, is the eddy-current control method.

Some authors have been made some researches enforce to find reliability NDT techniques to detect and sizing the micro root defects on FSW. Nevertheless, in conductivity materials, when high reliability is required as mentioned before, eddy current is the most adequate technique. However, eddy current (EC) flow can change not only due to defects, but also due to the variations of the probe lift-off. The high-sensitive lift-off effect of conventional EC probes introduces noises which mask the signal of small defects, making their detection difficult or even impossible. Moreover, the low sensitivity of common EC probes as well as other electronic issues may not enable to distinguish between defect and non defective conditions.

Catalin Mandache et al. [3] applied PEC in FSW, but even in the presence of significant size root defects the inspection accuracy is low. PEC variant has the capability to detect deeper defects, nevertheless micro-defects in FSW can hardly be detected.. Neil Goldfine et al. [4] have developed a MWM ${ }^{\circledR}$ probe which is able to detect some FSW defects, but only quite large (typically above 150 micron). Lamarre et al. [5] were among the first to apply phased array ultrasonic on FSW, and P. H. Johnston [6] highlighted difficulties in detecting the oxides alignment defects on the nugget of the FSW using this NDT technique.

M. Moles et al [7] used ultrasonic and eddy currents arrays for inspection of friction stir welds in aluminum. L .S. Rosado et al [8] used eddy current probe to detect the imperfections in the friction stir welding of metals. Li et al [9] have reported on multiple nondestructive testing methods on the FSW of AA 2219-T6. Hu et al [10] also employed a high-precision magnetic sensor to detect the weld defects in aluminium Friction stir welds.

In [11] a new NDT system is presented. The system is composed by a planar eddy currents probe (IOnic probe), electronic devices for signal generation, conditioning and conversion, automated mechanized scanning and analysis software. The IOnic system was developed mostly to be used for the micro-defects detection on aluminum solid state processed alloys by FSW. The experimental results in AA2024 welded by FSW clearly show that this system is able to detect imperfections around $50 \mu \mathrm{m}$, which contribute to increase the reliability on NDT of micro imperfections.

Therefore, the task of developing an eddy-current transducer capable of examining welded joints obtained by means of friction stir welding and the creation of eddy-current measuring system capable of detecting flaws in such materials seems to be crucial.

The purpose of this work is the detection of defects in welded FSW joints of aluminium alloys with the application of the eddy-current transducer and the development of the eddycurrent control procedure for such joints.

\section{Materials and methods}

The authors have developed a subminiature eddy-current transformer for local monitoring of the physical parameters in titanium-alloy plates and weld seams [11]. In contrast to existing sensors, it permits local measurements with sections measuring a few microns, to depths of the order of $5 \mathrm{~mm}$. The electrical conductivity of the alloy is directly measurable, and its distribution over the sample surface and thickness may be readily established. The eddycurrent method is based on the dependence of the current magnitude and distribution on the 
geometric and electromagnetic parameters of the sample and on the mutual position of the sensor and the sample. The basic informative parameter $\beta_{0}$ is a generalized characteristic of the object, the eddy-current sensor, and the frequency of the electromagnetic field [12]. We need to develop an adequate model of the response of a eddy-current transformers of plate type, which are sensitive to many variables and permit reproduction of the voltage hodograph at small values of $\beta_{0}$. We have plotted hodographs illustrating the influence of various parameters on the induced voltage on the basis of the proposed model $[13,14]$.

The eddy-current transformer is connected to the sound board of a computer with special software that controls the voltage in the transformers exciting winding and also reads the voltage at the measuring winding (in arbitrary units). On the basis of preliminary calibration, these readings are converted to values of the electrical conductivity. The exciting winding of the transducer consists of ten turns; its diameter is $0.12-0.13 \mathrm{~mm}$. The measuring winding consists of 130 turns and has a diameter of $0.05-0.08 \mathrm{~mm}$. To minimize the influence of the exciting winding on the recording signal, the circuit contains a compensation winding that is connected to the measuring winding in accordance with the well-known differential circuit. This consists of 20 turns. A copper wire with a $5 \mu \mathrm{m}$ thickness is used for winding turns. The turns are wound around a pyramidal core. The proposed shape of the core is favorable for the area of the magnetic field. The core is made of ferrite with an initial magnetic permeability of 500 .

Different transducers that are based on cores that have the same ratio of the base diagonal $(400 \mu \mathrm{m})$ and edge length $(1 \mathrm{~mm})$ were calibrated using samples with a well-known electric conductivity. The characteristics of the designed transducers allow one to efficiently localize the magnetic field within $2500 \mu^{2}$ and provide penetration of the magnetic field into the studied object at a depth of up to $5 \mathrm{~mm}$.

The eddy-current transducer (Fig. 1.) is a transformer with measuring (1), exciting (2), and compensation (3) windings and a magnetic circuit 4, which is located inside the cylindrical platform 5 with tracks that are cut on the external side for windings. The platform is impregnated with a compound 6 at a temperature of $200^{\circ} \mathrm{C}$ to prevent the disintegration of the windings when the ferrite screen 7 , which is intended for the localization of the electromagnetic field on the tested object, is put in place. From the outside the transducer is contained in a corundum washer 8 , which protects the core 4 from contacting the tested object.

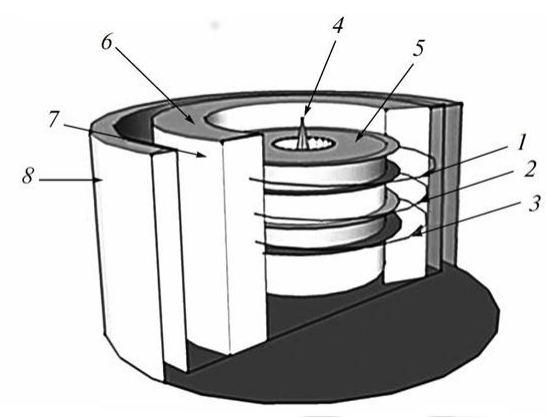

Fig. 1. A block diagram of the eddy-current transducer.

The measuring system, which is based on a miniature eddy-current transducer, operates as follows. The software of the personal computer controls the operation of the generator, which produces a train of rectangular voltage pulses with the repetition rate $f_{1}$ that is necessary for the operation of the eddy-current transducers. The voltage pulses are transmitted from the generator output to two series integrators. They are then directed to the input of the power amplifier. From the amplifier output the voltage pulses arrive at the 
exciting inductance coils of the eddy-current transducers. The difference of the output voltages of the measuring coils of the transducers contains information on the structural heterogeneities of the tested object that is located in the effective area of the eddy-current transducers. It is detected and amplified in a special microphone amplifier. The signal arrives at the amplitude detector after the transmission through two series high-quality low frequency filters and two series selective amplifiers. The signal is then transmitted through an analogto-digital converter to a personal computer. Due to the simultaneous control of the generated signal frequency at the exciting coil and the cut-off frequency of the filtering system and the selective amplification, the useful signal, which contains information on the electric conductivity distribution inside the object, in particular, on possible flaws of the object, is detected. The control program allows one to change the operating frequency of the measuring system so that the signal that is received from the measuring winding is reliably recorded.

\section{Result and discussion}

Defectoscopy was performed with the help of a transformer eddy current probe of the overhead type, field intensity of generating winding - $800 \mathrm{~A} / \mathrm{m}$. The measured characteristic is introduced voltage induced by the field of eddy currents arising in monitored object. The sensor calibration was performed before the beginning of the measuring operations, the calibration itself consisting in the definition of the input voltage from the defect-free section. The calibration was performed at the frequency of $700 \mathrm{~Hz}$. After that, the detector was moving over the scanned defective area, while the voltage input in the measuring winding of the transducer was being registered. The authors tried to determine the frequency that provided for the most input voltage deviation from the voltage value obtained at the defectfree section of the specimen. The voltage value corresponding to this frequency was viewed as a parameter, which behavior allowed inferring the presence of a defect. Scanning was carried out by moving of sensor across welds or across deforming area.

Sample No. 1: two aluminum-magnesium alloy slats connected by friction welding. Thickness of the slats was $8.3 \mathrm{~mm}$. Width of the weld was $2 \mathrm{~cm}$. Scanning was carried out by moving the sensor perpendicular to the joint line, measuring the distance from the calibration area to the measurement area.

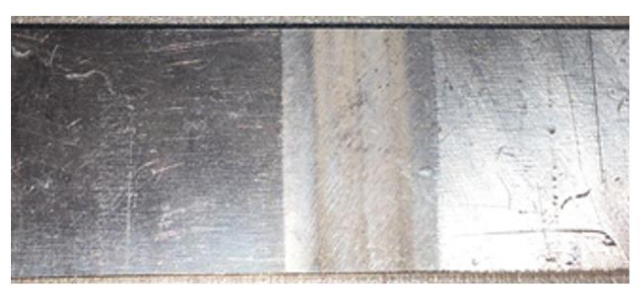

a

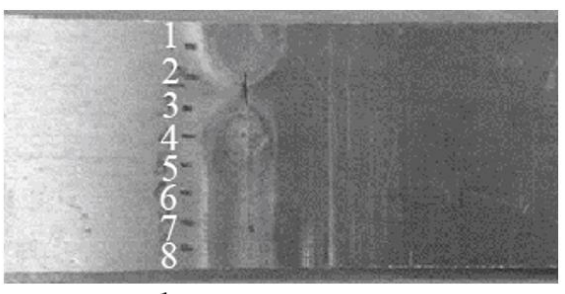

b

Fig. 2. Photo of Sample No. 1 and Sample No. 2 surface with marked scanned areas.

In the experiments with sample No. 1(Fig.2.a.), it was found that slats welded together by weld, give different values of introduced voltage that, obviously, indicates different materials of slats. The results of the experiment are presented in Fig.3. The boundaries of weld correspond to drop of the introduced voltage. At the boundaries of weld, it is possible to see sections of significant drop in the introduced voltage, corresponding to the boundaries of the weld. 


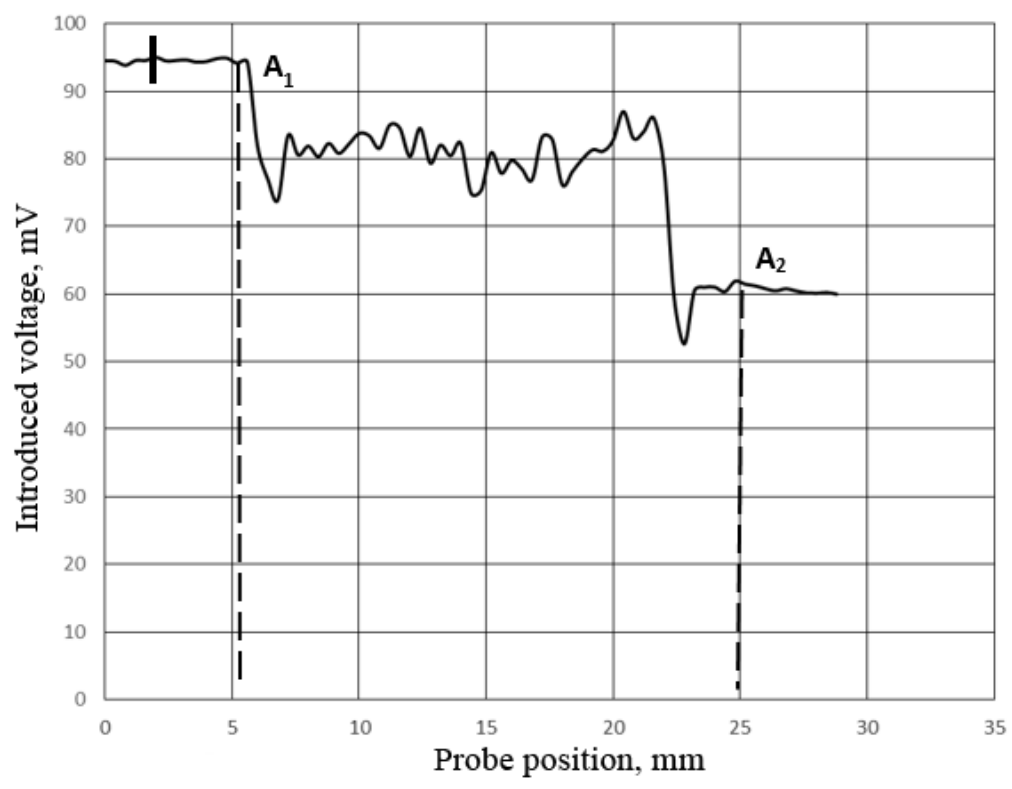

Fig. 3. Amount of the voltage introduced to measuring winding of the transducer in the area of a weld seam. Scanning frequency is $700 \mathrm{~Hz} . \mathrm{A}_{1}-\mathrm{A}_{2}$ are welding edges

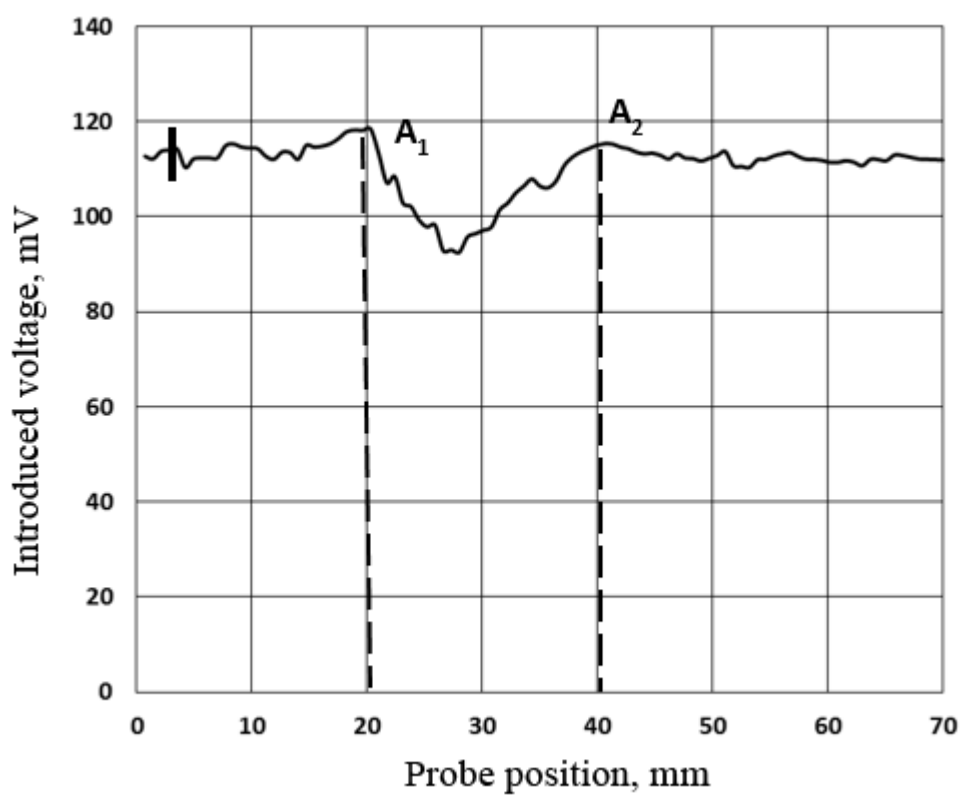

Fig. 4. a. Measurement results for the defective weld seam, Area $1 . A_{1}-A_{2}$ are welding edges, $\mathrm{C}$ is the location of the crack 


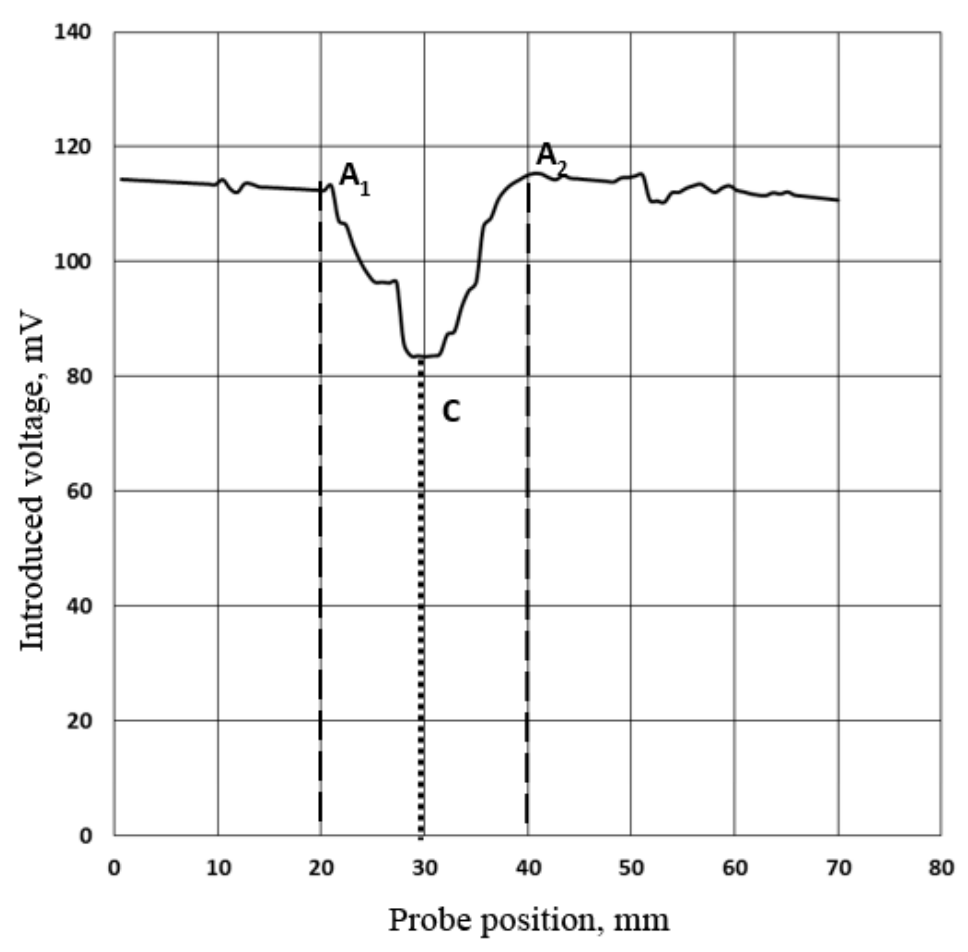

Fig. 4. b. Measurement results for the defective weld seam, Area $2 . A_{1}-A_{2}$ are welding edges, $\mathrm{C}$ is the location of the crack

Sample No. 2 (Fig. 2.b.) is a 5-mm-thick plate having technological flaws in the area of the welded joint (scanned sections are labelled with numbers 1, 2, 3, 4, 5, 6, 7, 8 and are presented in the picture). Scanning was performed by moving the sensor perpendicularly to the joint line, measuring the distance from the calibration area to the measurement area. In this sample, the scanning was performed by moving the sensor over the area of weld with the defects contained therein.

In the area 1, inside the sample, there was a cavity with diameter $2 \mathrm{~mm}$, the depth of occurrence from the scanning surface was $3 \mathrm{~mm}$. The cavity was fixed during scanning with

the use of an eddy current probe operating at a frequency of $700 \mathrm{~Hz}$ (Fig. 4.a.). The boundaries of weld correspond to the drop in introduced voltage. The dotted line indicates the defect area. The drop of voltage across the measuring winding of the probe in the defect area was $25 \mathrm{mV}$ compared to the calibration area and $7 \mathrm{mV}$ compared to the weld area

In areas 2 and 3, there was a surface crack on the welding joint. The width of the crack was $0.2 \mathrm{~mm}$. The surface crack was easily fixed by the probe at practically all frequencies: the change in the applied voltage was about $35 \mathrm{mV}$ compared to the calibration area and about $15 \mathrm{mV}$ compared to the weld area (Fig 4.b., 5.a.). 


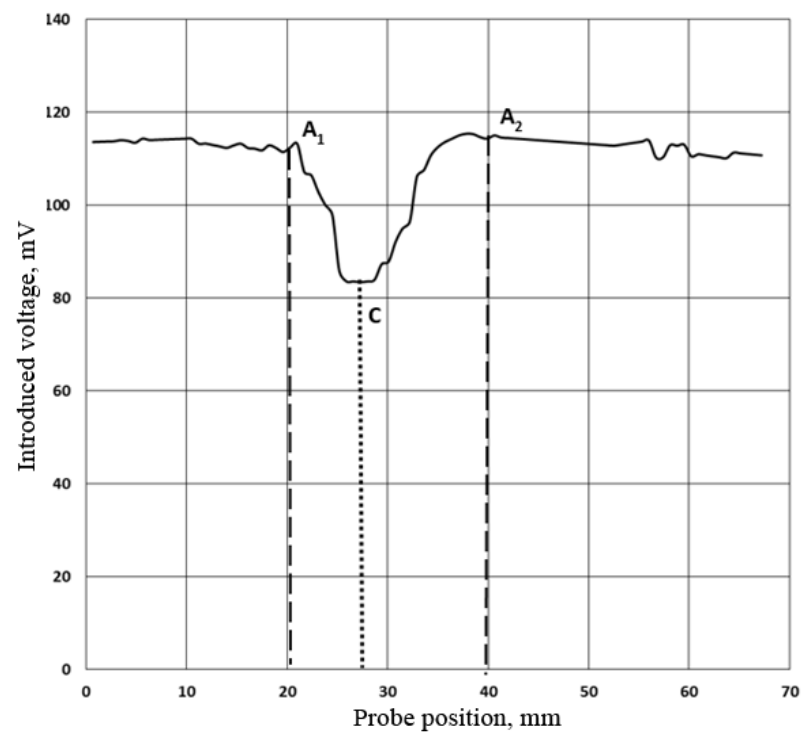

Fig. 5. a. Measurement results for the defective weld seam, Area 3. $A_{1}-A_{2}$ are welding edges, $\mathrm{C}$ is the location of the crack, $\mathrm{B}_{1}-\mathrm{B}_{2}$ are the cavity edges.

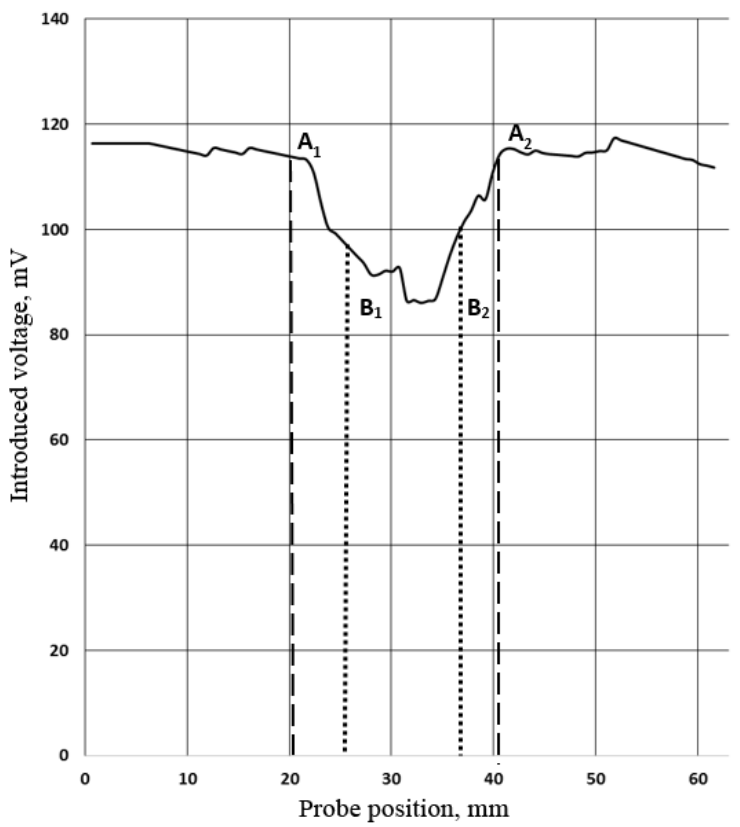

Fig. 5.b. Measurement results for the defective weld seam, Area 4. $A_{1}-A_{2}$ are welding edges, $\mathrm{C}$ is the location of the crack, $\mathrm{B}_{1}-\mathrm{B}_{2}$ are the cavity edges.

Area 4 is a milled internal cylindrical cavity located on the back of the slat, $4 \mathrm{~mm}$ in depth, $15 \mathrm{~mm}$ in diameter, with depth of $1 \mathrm{~mm}$ from the scanning surface, which is clearly visible on the probe (Fig. 5.b.). The change of introduced voltage in the area of the cavity is $30 \mathrm{mV}$ compared to the calibration area and $10 \mathrm{mV}$ compared to the weld area. 
Areas 5-8 were presented as a weld area, having internal cavities (holes) marked with characteristic dips at the edges of the joint. (Fig. 6.a-d). The drop in introduced voltage across the gaps was 5-10 $\mathrm{mV}$ compared to the neighbouring defect-free area of the sample. Since a similar pattern was observed in a sample No. 1 (Fig. 3.), it can be concluded that this feature is inherent to this type of welding.

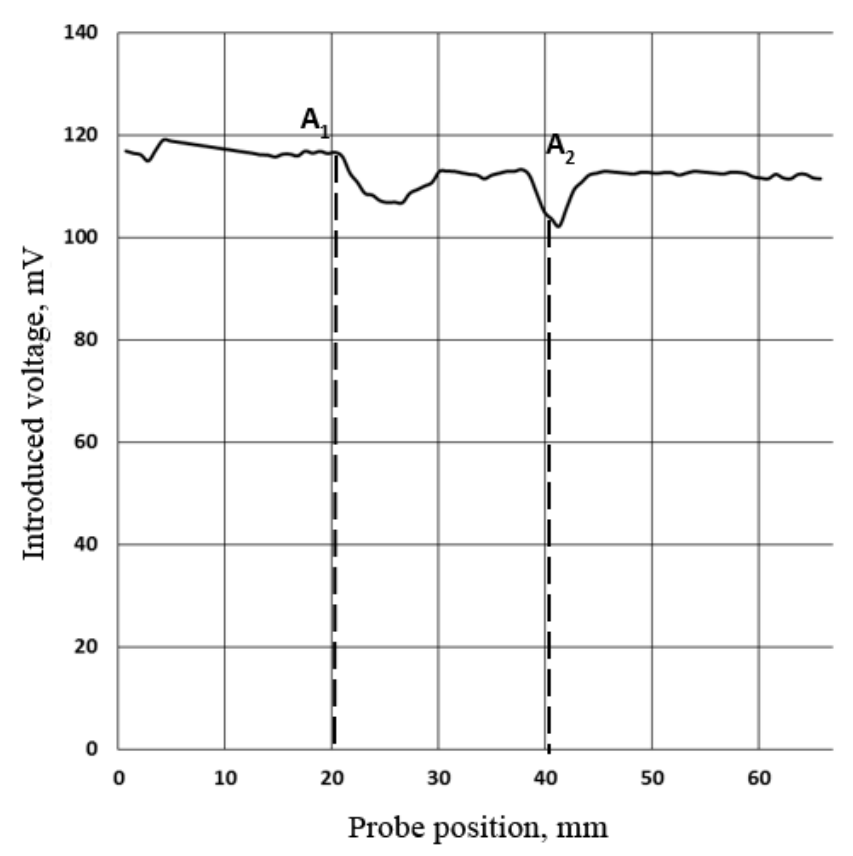

Fig. 6.a. Measurement results for the defective weld seam, Area 5. $A_{1}-A_{2}$ are welding edges

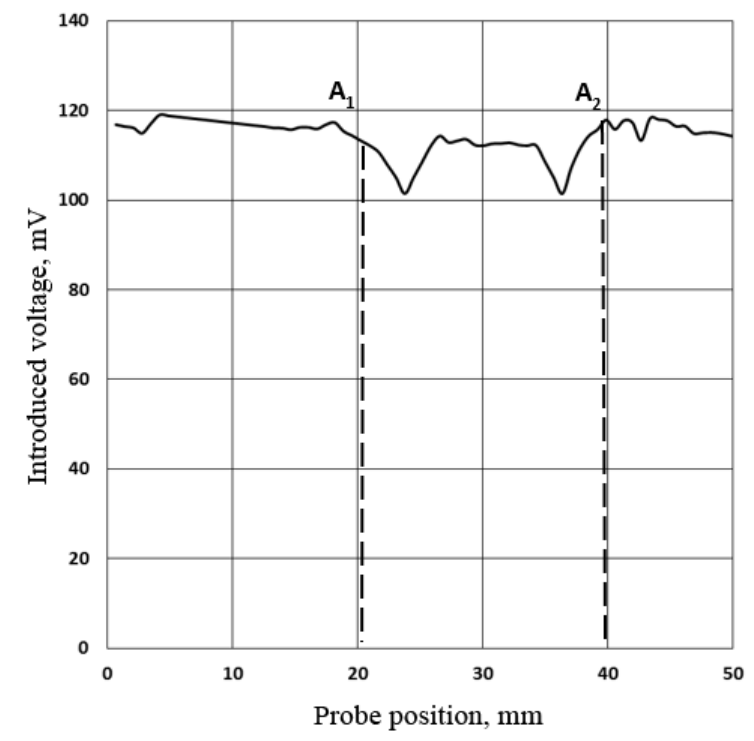

Fig. 6.b. Measurement results for the defective weld seam, Area $6 . A_{1}-A_{2}$ are welding edges 


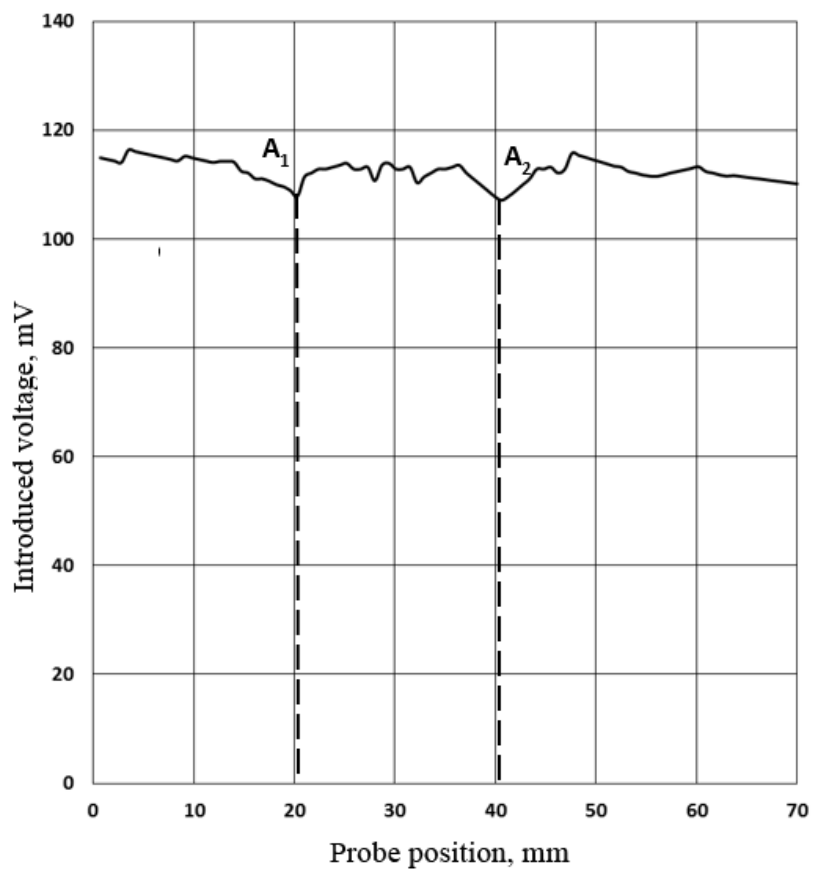

Fig. 6.c. Measurement results for the defective weld seam, Area 7. $A_{1}-A_{2}$ are welding edges

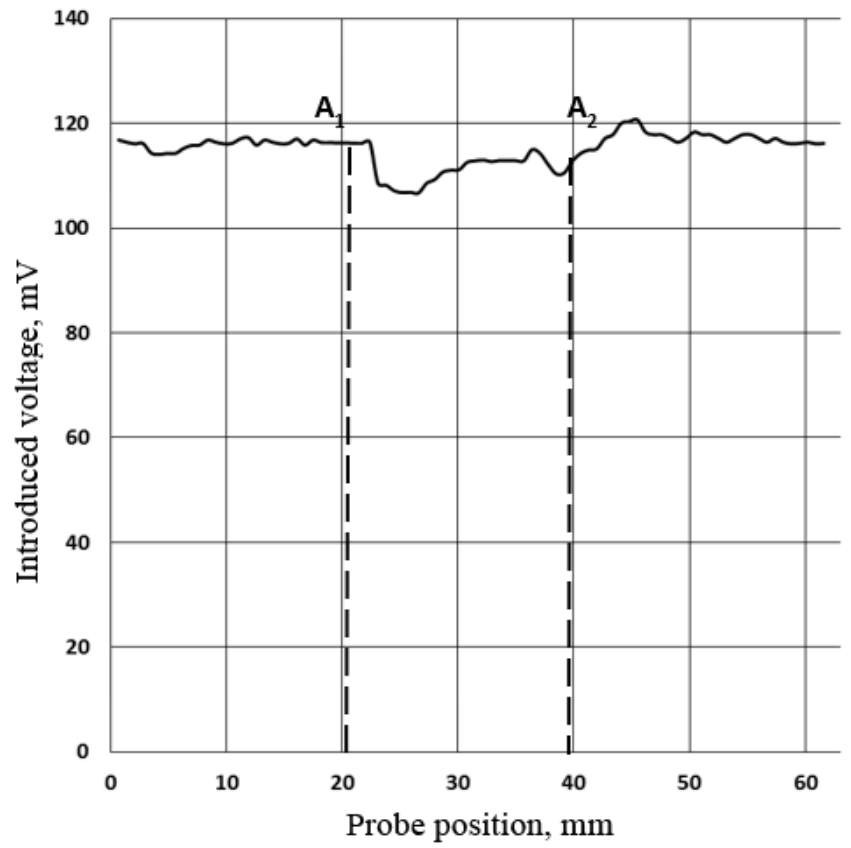

Fig. 6.d. Measurement results for the defective weld seam, Areas $8 . A_{1}-A_{2}$ are welding edges 


\section{Conclusion}

We worked out a measuring system aimed at the examination of plates made of aluminiummagnesium alloys joint with the help of friction stir welding method. The conducted research demonstrates the potential of the eddy-current control method applied to search for midget flaws in the welded joints. Subminiaturized eddy-current transducers, constructed on the basis of pyramid-shaped cores, allow effectively detecting cracks and cavities having the diameter from 0.2 to $2 \mathrm{~mm}$ located at the depth of up to $3 \mathrm{~mm}$. In addition, we demonstrated the possibility to control the areas of weld seam edges.

\section{References}

1. S. Mironov, Physical Mesomechanics, 17, 103 (2014)

2. V Rubtsov, E Kolubaev, S Tarasov, P Vasilyev, V Bakshaev, AIP Conf. Proc, 1623, 535 (2014)

3. C Mandache, L Dubourg A Merati, Materials Evaluation, 4, 382 (2008)

4. D Grundy, V Zilberstein, N Goldfine, Proc. of 7th International Conference on Trends in Welding Research, 1, 227 (2005)

5. A Lamarre, M Michael, AIP Conference Proceedings, 509, 1333 (2000)

6. P Johnston, 35th Annual Review of Progress in Quantitative Nondestructive Evaluation, 1, 1 (2008)

7. M Moles, A Lamarre, O Dupuis, 16th World Conference on NDT, 84, 50 (2004)

8. L Rosado, T Santos, M Piedade, P Ramos, P Vilaca, Measurement: Journal of the International Measurement Confederation, 43(8), 1021 (2010)

9. B Li, Y Shen, W Hu, Materials and Design, 32(4), 2073 (2011)

10. B Hu, R Yu, H Zou, NDT\&E International, 47, 66 (2012)

11. V Pedro, G Telmo, R Luís, M Rosa, The 12th International Conference of the Slovenian Society for Non-Destructive Testing »Application of Contemporary Non-Destructive Testing in Engineering», 1, 329 (2013)

12. S F Dmitriev, V N Malikov, A M Sagalakov, M A Davydchenko, Proceedings of 2015 International Conference on Mechanical Engineering, Automation and Control Systems, MEACS 2015, 1, 1 (2016)

13. V N Malikov, S F Dmitiev, Russ. Eng. Res., 8, 32 (2016)

14. S F Dmitriev, V N Malikov, A M Sagalakov, Welding International, 31(8), 608 (2017) 